\title{
The equal split-off set for NTU-games
}

Citation for published version (APA):

Dietzenbacher, B., \& Yanovskaya, E. (2022). The equal split-off set for NTU-games. Maastricht University, Graduate School of Business and Economics. GSBE Research Memoranda No. 002 https://doi.org/10.26481/umagsb.2022002

Document status and date:

Published: 21/02/2022

DOI:

10.26481/umagsb.2022002

Document Version:

Publisher's PDF, also known as Version of record

\section{Please check the document version of this publication:}

- A submitted manuscript is the version of the article upon submission and before peer-review. There can be important differences between the submitted version and the official published version of record.

People interested in the research are advised to contact the author for the final version of the publication, or visit the DOI to the publisher's website.

- The final author version and the galley proof are versions of the publication after peer review.

- The final published version features the final layout of the paper including the volume, issue and page numbers.

Link to publication

\footnotetext{
General rights rights.

- You may freely distribute the URL identifying the publication in the public portal. please follow below link for the End User Agreement:

www.umlib.nl/taverne-license

Take down policy

If you believe that this document breaches copyright please contact us at:

repository@maastrichtuniversity.nl

providing details and we will investigate your claim.
}

Copyright and moral rights for the publications made accessible in the public portal are retained by the authors and/or other copyright owners and it is a condition of accessing publications that users recognise and abide by the legal requirements associated with these

- Users may download and print one copy of any publication from the public portal for the purpose of private study or research.

- You may not further distribute the material or use it for any profit-making activity or commercial gain

If the publication is distributed under the terms of Article $25 \mathrm{fa}$ of the Dutch Copyright Act, indicated by the "Taverne" license above, 


\section{Maastricht University}

Bas Dietzenbacher,

Elena Yanovskaya

The equal split-off set for NTUgames

$\mathrm{RM} / 22 / 002$

ISSN: $2666-8807$

\section{GSBE}

Maastricht University School of Business and Economics

Graduate School of Business and Economics

P.O Box 616

NL-6200 MD Maastricht

The Netherlands 


\title{
The equal split-off set for NTU-games
}

\author{
Bas Dietzenbacher* Elena Yanovskaya ${ }^{\dagger}$
}

February 17, 2022

\begin{abstract}
This paper introduces and studies the equal split-off set for cooperative games with nontransferable utility. We illustrate the new solution for the famous Roth-Shafer examples and present two axiomatic characterizations based on different consistency properties on the class of exact partition games, i.e. the class of games where it intersects the core. Moreover, we provide explicit expressions for the class of bargaining problems and the class of bankruptcy problems.
\end{abstract}

Keywords: nontransferable utility games; equal split-off set; exact partition games; consistency

JEL classification: $\mathrm{C} 71$

\section{Introduction}

Nontransferable utility games arise when players in a cooperative game face the problem of allocating joint profits while having nonlinear utility functions over money. Also situations where the underlying infinitely divisible endowment is not of a monetary nature are accommodated. The opportunities of coalitions are represented by a set of attainable utility payoff allocations and the issue is to select a payoff allocation for the grand coalition while taking these opportunities into account.

We focus on egalitarianism in the context of nontransferable utility games. Since egalitarianism is a doctrine that favors the idea of equality, this requires the assumption that utility is not only comparable in an intrapersonal way, but also in an interpersonal way. In other words, we assume that utility is normalized in such a way that equating utilities for different players has a meaningful interpretation. This was also implied by the approach of Kalai and Samet (1985), who introduced and characterized an egalitarian solution for nontransferable utility games which recursively assigns equal payoffs to members of coalitions in

\footnotetext{
*Department of Quantitative Economics, Maastricht University, Maastricht, The Netherlands. E-mail: b.dietzenbacher@maastrichtuniversity.nl

$\dagger$ International Laboratory of Game Theory and Decision Making, HSE University, St. Petersburg, Russia. E-mail: eyanovskaya@hse.ru
} 
the form of dividends. Samet (1985) and De Clippel et al. (2004) provided other axiomatic characterizations of this solution. On the class of transferable utility games, the Kalai and Samet (1985) solution coincides with the Shapley (1953) value. On the class of bargaining problems (cf. Nash 1950), it coincides with the egalitarian Kalai (1977) solution.

We introduce and study the equal split-off set for nontransferable games. Inspired by the computational algorithm of the egalitarian Dutta and Ray (1989) solution, Branzei et al. (2006) introduced the equal split-off set for transferable utility games. We generalize that definition in the following way. Imagine that the players of a nontransferable utility game believe in equality as a social goal. We start proposing the maximal attainable equal payoff allocation for the grand coalition. Some coalitions may complain, because their maximal attainable equal payoff allocation may give them higher payoffs. We select one coalition with the highest complaint, assign its members their maximal attainable equal payoffs, and let them leave the game. The remaining players now face a reduced game in which coalitions are able to attain all original payoff allocations in cooperation with the departed players. Again, the maximal attainable equal payoff allocation is proposed, but coalitions may complain. The process is repeated until all players have left the game, leading to a payoff allocation for the grand coalition. The equal split-off set consists of all payoff allocations generated by this procedure.

We illustrate the equal split-off set for nontransferable utility games using the famous Roth-Shafer examples and compare it with the Kalai and Samet (1985) solution for these games. We show that the equal split-off set intersects the core if and only if the underlying game is an exact partition game, i.e. there exists a core element for which all richest players, all richest and second richest players, and so on, are able to obtain their payoffs jointly by themselves. This generalizes the class of transferable utility games introduced by Llerena and Mauri (2017) and the corresponding result of Dietzenbacher and Yanovskaya (2021). On the class of exact partition games, we present two axiomatic characterizations of the equal split-off set based on weak versions of consistency properties that were employed by Peleg (1985) and Tadenuma (1992) to characterize the core. It turns out that nontransferable utility games induced by bargaining problems and bankruptcy problems are exact partition games. On the class of bargaining problems, the equal split-off set coincides with the Kalai (1977) solution. On the class of bankruptcy problems with nontransferable utility, the equal split-off set coincides with the constrained Kalai solution (cf. Albizuri et al. 2020).

This paper is organized in the following way. Section 2 presents preliminary notions and notations for nontransferable utility games. Section 3 introduces the equal split-off set as a solution for all nontransferable utility games and presents some elementary results. Section 4 introduces the class of exact partition games, shows that this class consists of all games where the equal split-off set intersects the core, and presents two axiomatic characterizations based on different consistency properties. Section 5 illustrates the equal split-off set for the class of bargaining problems and the class of bankruptcy problems with nontransferable utility. 


\section{Preliminaries}

Let $N$ be a nonempty and finite set. Denote $2^{N}=\{S \mid S \subseteq N\}$. An ordered partition of $N$ is an ordered set $\left\{T_{1}, \ldots, T_{m}\right\} \subseteq 2^{N} \backslash\{\emptyset\}$ such that $\bigcup_{k=1}^{m} T_{k}=N$ and $T_{k} \cap T_{\ell}=\emptyset$ for all $k, \ell \in\{1, \ldots, m\}$ with $k \neq \ell$. Let $e \in \mathbb{R}_{+}^{N}$ denote the vector of all ones, i.e. $e_{i}=1$ for all $i \in N$. For all $x, y \in \mathbb{R}_{+}^{N}, x \leq y$ denotes $x_{i} \leq y_{i}$ for all $i \in N$, and $x<y$ denotes $x_{i}<y_{i}$ for all $i \in N$. For all $x \in \mathbb{R}_{+}^{N}$ and all $S \in 2^{N}, x_{S} \in \mathbb{R}_{+}^{S}$ denotes $x_{S}=\left(x_{i}\right)_{i \in S}$. For all $A \subseteq \mathbb{R}_{+}^{N}$,

- the weak Pareto set is $\mathrm{WP}(A)=\{x \in A \mid \neg \exists y \in A: y>x\}$;

- the strong Pareto set is $\operatorname{SP}(A)=\{x \in A \mid \neg \exists y \in A: y \geq x, y \neq x\}$.

Note that $\mathrm{SP}(A) \subseteq \mathrm{WP}(A)$.

A nontransferable utility game is a pair $(N, V)$ where $N$ is a nonempty and finite set of players and $V$ assigns to each coalition $S \in 2^{N} \backslash\{\emptyset\}$ a set of attainable payoff allocations $V(S) \subseteq \mathbb{R}_{+}^{S}$ such that

- $V(S)$ is nonempty, closed, and bounded;

- $V(S)$ is comprehensive, i.e. $\left\{y_{S} \in \mathbb{R}_{+}^{S} \mid y_{S} \leq x_{S}\right\} \subseteq V(S)$ for all $x_{S} \in V(S)$;

- $(N, V)$ is monotonic, i.e. $V(S) \subseteq\left\{x_{S} \mid x_{T} \in V(T)\right\}$ for all $S, T \in 2^{N} \backslash\{\emptyset\}$ with $S \subseteq T$.

The nonnegativity condition on the attainable payoff allocations was also asssumed by e.g. Asscher (1976), Asscher (1977), Greenberg (1985), and Lejano (2011). This convenient assumption is without loss of generality, i.e. our analysis is invariant under symmetric positive transformations of utility. The nonemptiness, closedness, boundedness, and comprehensiveness conditions are standard. The monotonicity condition was also assumed by e.g. Otten et al. (1998) and Hendrickx et al. (2002). Note that we do not assume that the sets of attainable payoff allocations are convex in order to allow for utility functions that are not necessarily of the Von Neumann-Morgenstern type. In line with Kalai and Samet (1985), we assume that utility is normalized in such a way that it is interpersonally comparable.

Let $\Gamma_{\text {all }}$ denote the class of all NTU-games. A solution $\sigma$ on a class of games $\Gamma \subseteq \Gamma_{\text {all }}$ assigns to each $(N, V) \in \Gamma$ a set of payoff allocations $\sigma(N, V) \subseteq V(N)$. Throughout this paper, $\Gamma$ is the generic notation for a class of games and $\sigma$ is the generic notation for a solution on $\Gamma$. The core is the solution on $\Gamma_{\text {all }}$ that assigns to each $(N, V) \in \Gamma_{\text {all }}$ the set of payoff allocations

$$
C(N, V)=\left\{x \in V(N) \mid \forall S \in 2^{N} \backslash\{\emptyset\} \neg \exists y_{S} \in V(S): y_{S}>x_{S}\right\}
$$




\section{The equal split-off set}

In this section, we introduce the equal split-off set as a solution for all nontransferable utility games and present some elementary results. The equal split-off set for transferable utility games introduced by Branzei et al. (2006) is based on the computational algorithm of the egalitarian Dutta and Ray (1989) solution. We generalize this solution to nontransferable utility games in the following way. Consider an arbitrary nontransferable utility game for which we face the problem of selecting payoff allocations for the grand coalition. One of the coalitions with maximal attainable equal payoff allocation is selected and the members leave with these payoffs. The remaining players determine the attainable payoff allocations for each subgroup in coalition with the departed players. One of the subgroups with maximal attainable equal payoff allocation is selected and the members leave with these payoffs. This process continues and results in a payoff allocation for the players. The equal split-off set consists of all payoff allocations generated by this procedure.

Definition 1 (Equal split-off set)

Let $(N, V) \in \Gamma_{\text {all. }}$. Define $N_{0}=N, V_{0}=V$, and $T_{0}=\emptyset$. The equal split-off set $\operatorname{ESOS}(N, V)$ consists of all payoff allocations $x \in \mathbb{R}_{+}^{N}$ for which there exists an ordered partition $\left\{T_{1}, \ldots, T_{m}\right\}$ of $N$ such that for all $k \in\{1, \ldots, m\}$,

$$
\begin{aligned}
T_{k} & \in \underset{S \in 2^{N_{k}} \backslash\{\emptyset\}}{\operatorname{argmax}} \max \left\{t \in \mathbb{R}_{+} \mid t e_{S} \in V_{k}(S)\right\} \\
\text { and } \quad x_{i} & =\max _{S \in 2^{N_{k}} \backslash\{\emptyset\}} \max \left\{t \in \mathbb{R}_{+} \mid t e_{S} \in V_{k}(S)\right\} \text { for all } i \in T_{k},
\end{aligned}
$$

where $\left(N_{k}, V_{k}\right)$ is the game defined by $N_{k}=N_{k-1} \backslash T_{k-1}$ and

$$
V_{k}(S)=\left\{y_{S} \in \mathbb{R}_{+}^{S} \mid\left(y_{S}, x_{T_{k-1}}\right) \in V_{k-1}\left(S \cup T_{k-1}\right)\right\} \text { for all } S \in 2^{N_{k}} .
$$

Note that the equal split-off set is well-defined and nonempty for all nontransferable utility games. We illustrate this new solution by means of the examples introduced by Roth (1980) and Shafer (1980). These examples initiated an interesting and extensive discussion on the interpretation of solutions for nontransferable utility games. For details, we refer to Harsanyi (1980), Aumann (1985b), Hart (1985b), Roth (1986), and Aumann (1986). Along the lines of this discussion, we compare the equal split-off set with the egalitarian Kalai and Samet (1985) solution. 
Example 1 (cf. Roth 1980)

Let $\left(N, V_{p}\right)$ with $N=\{1,2,3\}$ and $p \in\left[0, \frac{1}{2}\right]$ be the game given by

$$
V_{p}(S)= \begin{cases}\left\{x_{i} \in \mathbb{R}_{+}^{\{i\}} \mid x_{i} \leq 0\right\} & \text { if } S=\{i\} \text { and } i \in N ; \\ \left\{x_{\{1,2\}} \in \mathbb{R}_{+}^{\{1,2\}} \mid\left(x_{1}, x_{2}\right) \leq\left(\frac{1}{2}, \frac{1}{2}\right)\right\} & \text { if } S=\{1,2\} ; \\ \left\{x_{\{1,3\}} \in \mathbb{R}_{+}^{\{1,3\}} \mid\left(x_{1}, x_{3}\right) \leq(p, 1-p)\right\} & \text { if } S=\{1,3\} ; \\ \left\{x_{\{2,3\}} \in \mathbb{R}_{+}^{\{2,3\}} \mid\left(x_{2}, x_{3}\right) \leq(p, 1-p)\right\} & \text { if } S=\{2,3\} ; \\ \operatorname{comp}\left(\operatorname{conv}\left(\left\{\left(\frac{1}{2}, \frac{1}{2}, 0\right),(p, 0,1-p),(0, p, 1-p)\right\}\right)\right) & \text { if } S=N .\end{cases}
$$

Here, comp denotes the comprehensive hull and conv denotes the convex hull, i.e. the smallest containing comprehensive set and the smallest containing convex set, respectively.

If $p<\frac{1}{2}$, the equal split-off set is $\operatorname{ESOS}\left(N, V_{p}\right)=\left\{\left(\frac{1}{2}, \frac{1}{2}, 0\right)\right\}$ corresponding to ordered partition $\{\{1,2\},\{3\}\}$ and the Kalai and Samet (1985) solution is $\left(\frac{1}{2}-\frac{1}{3} p, \frac{1}{2}-\frac{1}{3} p, \frac{2}{3} p\right)$. Note that, in contrast to the Kalai and Samet (1985) solution, the equal split-off set assigns the unique core element to this game. Moreover, Roth (1980) claims that $\left(\frac{1}{2}, \frac{1}{2}, 0\right)$ is the unique outcome of this game consistent with the hypothesis that the players are rational utility maximizers, because this outcome is strictly preferred by both players 1 and 2 over all other feasible outcomes, and it can be achieved without the cooperation of player 3 .

If $p=\frac{1}{2}$, the game is completely symmetric with respect to the players and it is no longer the case that cooperation with player 3 offers strictly less to players 1 or 2 than cooperation with one another. The equal split-off set is $\operatorname{ESOS}\left(N, V_{\frac{1}{2}}\right)=\left\{\left(\frac{1}{2}, \frac{1}{2}, 0\right),\left(\frac{1}{2}, 0, \frac{1}{2}\right),\left(0, \frac{1}{2}, \frac{1}{2}\right)\right\}$ corresponding to ordered partitions $\{\{1,2\},\{3\}\},\{\{1,3\},\{2\}\}$, and $\{\{2,3\},\{1\}\}$. The Kalai and Samet (1985) solution is $\left(\frac{1}{3}, \frac{1}{3}, \frac{1}{3}\right)$. Note that the equal split-off set coincides with the core in this case, whereas the Kalai and Samet (1985) solution is not in the core.

Example 2 (cf. Shafer 1980 and Hart and Kurz 1983)

Let $\left(N, V_{\varepsilon}\right)$ with $N=\{1,2,3\}$ and $\varepsilon \in\left[0, \frac{1}{5}\right]$ be the game given by

$$
V_{\varepsilon}(S)= \begin{cases}\left\{x_{i} \in \mathbb{R}_{+}^{\{i\}} \mid x_{i} \leq 0\right\} & \text { if } S=\{i\} \text { and } i \in\{1,2\} ; \\ \left\{x_{3} \in \mathbb{R}_{+}^{\{3\}} \mid x_{3} \leq \varepsilon\right\} & \text { if } S=\{3\} ; \\ \left\{x_{\{1,2\}} \in \mathbb{R}_{+}^{\{1,2\}} \mid x_{1}+x_{2} \leq 1-\varepsilon\right\} & \text { if } S=\{1,2\} ; \\ \left\{x_{\{1,3\}} \in \mathbb{R}_{+}^{\{1,3\}} \mid x_{1} \leq \varepsilon, x_{1}+x_{3} \leq \frac{1}{2}+\frac{1}{2} \varepsilon\right\} & \text { if } S=\{1,3\} ; \\ \left\{x_{\{2,3\}} \in \mathbb{R}_{+}^{\{2,3\}} \mid x_{2} \leq \varepsilon, x_{2}+x_{3} \leq \frac{1}{2}+\frac{1}{2} \varepsilon\right\} & \text { if } S=\{2,3\} ; \\ \left\{x \in \mathbb{R}_{+}^{N} \mid x_{1}+x_{2}+x_{3} \leq 1\right\} & \text { if } S=N .\end{cases}
$$

The equal split-off set is $\operatorname{ESOS}\left(N, V_{\varepsilon}\right)=\left\{\left(\frac{1}{2}-\frac{1}{2} \varepsilon, \frac{1}{2}-\frac{1}{2} \varepsilon, \varepsilon\right)\right\}$ corresponding to ordered partition $\{\{1,2\},\{3\}\}$ and the Kalai and Samet (1985) solution is $\left(\frac{1}{2}-\frac{5}{6} \varepsilon, \frac{1}{2}-\frac{5}{6} \varepsilon, \frac{5}{3} \varepsilon\right)$. Note that, in contrast to the Kalai and Samet (1985) solution, the equal split-off set assigns a core element to this game. 
Let $(N, V)$ be a game. Note that for each $x \in \operatorname{ESOS}(N, V)$ with corresponding ordered partition $\left\{T_{1}, \ldots, T_{m}\right\}$ the following holds:

- $x_{i}=x_{j}$ for all $i, j \in T_{k}$ with $k \in\{1, \ldots, m\}$;

- $x_{\bigcup_{\ell=1}^{k} T_{\ell}} \in \mathrm{WP}\left(V\left(\bigcup_{\ell=1}^{k} T_{\ell}\right)\right)$ for all $k \in\{1, \ldots, m\}$.

In contrast to transferable utility games, equal split-off set allocations make players not necessarily leave with their payoffs in nonincreasing order, i.e. it does not generally hold that $x_{i} \geq x_{j}$ for all $i \in T_{k}$ and $j \in T_{\ell}$ with $k, \ell \in\{1, \ldots, m\}$ and $k \leq \ell$. This is shown by the following example.

\section{Example 3}

Let $(N, V)$ with $N=\{1,2\}$ be the game given by

$$
V(S)= \begin{cases}\left\{x_{1} \in \mathbb{R}_{+}^{\{1\}} \mid x_{1} \leq 0\right\} & \text { if } S=\{1\} ; \\ \left\{x_{2} \in \mathbb{R}_{+}^{\{2\}} \mid x_{2} \leq 1\right\} & \text { if } S=\{2\} ; \\ \left\{x \in \mathbb{R}_{+}^{N} \mid\left(x_{1}, x_{2}\right) \leq(2,1)\right\} & \text { if } S=N .\end{cases}
$$

The equal split-off set is $\operatorname{ESOS}(N, V)=\{(2,1),(1,1)\}$ corresponding to ordered partitions $\{\{2\},\{1\}\}$ and $\{\{1,2\}\}$. Note that both equal split-off set allocations belong to the core of this game. For the allocation $(2,1)$, the payoff to player 2 is assigned first, while the higher payoff to player 1 is assigned last.

Observations change significantly if we slightly restrict the domain of nontransferable utility games. Let $\widetilde{\Gamma}_{\text {all }}$ denote the class of all NTU-games $(N, V)$ where for all $S \in 2^{N} \backslash\{\emptyset\}$,

- $V(S)$ is nonleveled, i.e. $\mathrm{SP}(V(S))=\mathrm{WP}(V(S))$.

Note that all nontransferable utility games in $\Gamma_{\text {all }}$ can be approximated by games in $\widetilde{\Gamma}_{\text {all }}$. The nonlevelness condition was also assumed by e.g. Aumann (1985a), Hart (1985a), Peleg (1985), Tadenuma (1992), and Hart and Mas-Colell (1996).

Lemma 1 (cf. Yanovskaya 2010)

Let $(N, V) \in \widetilde{\Gamma}_{\text {all }}$ and let $x \in \operatorname{ESOS}(N, V)$ with corresponding ordered partition $\left\{T_{1}, \ldots, T_{m}\right\}$. Then $x_{i} \geq x_{j}$ for all $i \in T_{k}$ and $j \in T_{k+1}$ with $k \in\{1, \ldots, m-1\}$.

Let $(N, V) \in \widetilde{\Gamma}_{\text {all }}$. For all $x \in \mathbb{R}_{+}^{N}$, we define $R_{0}^{x}=\emptyset$ and for all $k \in \mathbb{N}$,

$$
R_{k}^{x}=\left\{i \in N \mid \forall j \in N \backslash R_{k-1}^{x}: x_{j} \leq x_{i}\right\} \quad \text { and } \quad a_{k}^{x}=x_{i} \text { for all } i \in R_{k}^{x} \backslash R_{k-1}^{x} .
$$

Note that $R_{k-1}^{x} \subseteq R_{k}^{x}$ for all $k \in \mathbb{N}$ and $R_{|N|}^{x}=N$. Coalition $R_{1}^{x} \in 2^{N} \backslash\{\emptyset\}$ consists of the richest players in $x$, coalition $R_{2}^{x} \in 2^{N} \backslash\{\emptyset\}$ consists of the richest and second richest players in $x$, and so on. 
Lemma 1 implies that for each $x \in \operatorname{ESOS}(N, V)$ the following holds:

- $x_{R_{k}^{x}} \in \mathrm{WP}\left(V\left(R_{k}^{x}\right)\right)$ for all $k \in \mathbb{N}$.

In Example 3 the equal split-off set consists of multiple core allocations. Another consequence of assuming nonleveled attainable sets of payoff allocations is that the equal split-off set is single-valued when it intersects the core.

\section{Lemma 2}

Let $(N, V) \in \widetilde{\Gamma}_{\text {all }}$. If $\operatorname{ESOS}(N, V) \cap C(N, V) \neq \emptyset$, then $|\operatorname{ESOS}(N, V)|=1$.

Proof. Let $x \in \operatorname{ESOS}(N, V) \cap C(N, V)$. We show that for all $k \in \mathbb{N}$ with $R_{k-1}^{x} \neq N$,

$$
R_{k}^{x} \backslash R_{k-1}^{x}=\bigcup \underset{S \in 2^{N \backslash R_{k-1}^{x} \backslash\{\emptyset\}}}{\operatorname{argmax}} \max \left\{t \in \mathbb{R}_{+} \mid\left(t e_{S}, x_{R_{k-1}^{x}}\right) \in V\left(S \cup R_{k-1}^{x}\right)\right\} .
$$

Let $k \in \mathbb{N}$ be such that $R_{k-1}^{x} \neq N$. Note that $x_{i} \leq a_{k}^{x}$ for all $i \in N \backslash R_{k-1}^{x}$. Suppose for the sake of contradiction that there exist

$$
T \in \underset{S \in 2^{N \backslash R_{k-1}^{x}} \backslash\{\emptyset\}}{\operatorname{argmax}} \max \left\{t \in \mathbb{R}_{+} \mid\left(t e_{S}, x_{R_{k-1}^{x}}\right) \in V\left(S \cup R_{k-1}^{x}\right)\right\} \quad \text { and } \quad i \in T
$$

such that $x_{i}<a_{k}^{x}$. By comprehensiveness, $x_{T \cup R_{k-1}^{x}} \in V\left(T \cup R_{k-1}^{x}\right) \backslash \mathrm{SP}\left(V\left(T \cup R_{k-1}^{x}\right)\right)$. By nonlevelness, $x_{T \cup R_{k-1}^{x}} \in V\left(T \cup R_{k-1}^{x}\right) \backslash \mathrm{WP}\left(V\left(T \cup R_{k-1}^{x}\right)\right)$. This contradicts that $x \in$ $C(N, V)$.

\section{Exact partition games}

In this section, we introduce the class of exact partition games, we show that this class consists of all games where the equal split-off set intersects the core, and we present two axiomatic characterizations based on different consistency properties. Llerena and Mauri (2017) introduced exact partition games in the transferable utility context. We generalize this definition to nontransferable utility games. A game is an exact partition game if there exists a core allocation for which all richest players, all richest and second richest players, and so on, are able to obtain their payoffs jointly by themselves.

Definition 2 (Exact partition games)

A game $(N, V) \in \widetilde{\Gamma}_{\text {all }}$ is an exact partition game if there exists $x \in C(N, V)$ such that $x_{R_{k}^{x}} \in V\left(R_{k}^{x}\right)$ for all $k \in \mathbb{N}$. 


\section{Example 4}

Let $(N, V)$ with $N=\{1,2,3\}$ be the game given by

$$
V(S)= \begin{cases}\left\{x_{1} \in \mathbb{R}_{+}^{\{1\}} \mid x_{1} \leq 6\right\} & \text { if } S=\{1\} ; \\ \left\{x_{i} \in \mathbb{R}_{+}^{\{i\}} \mid x_{i} \leq 0\right\} & \text { if } S=\{i\} \text { and } i \in\{2,3\} ; \\ \left\{x_{\{1,2\}} \in \mathbb{R}_{+}^{\{1,2\}} \mid \min \left\{2 x_{1}+x_{2}, x_{1}+2 x_{2}\right\} \leq 12\right\} & \text { if } S=\{1,2\} ; \\ \left\{x_{S} \in \mathbb{R}_{+}^{S} \mid x_{S} \leq 0_{S}\right\} & \text { if } S \in\{\{1,3\},\{2,3\}\} ; \\ \left\{x \in \mathbb{R}_{+}^{N} \mid \sum_{i \in N} x_{i} \leq 10\right\} & \text { if } S=N .\end{cases}
$$

The equal split-off set is $\operatorname{ESOS}(N, V)=\{(6,3,1)\}$ corresponding to ordered partition $\{\{1\},\{2\},\{3\}\}$. Denote $x=(6,3,1)$. Then $x \in C(N, V)$. Moreover, $R_{1}^{x}=\{1\}, x_{1} \in V(\{1\})$, $R_{2}^{x}=\{1,2\}, x_{\{1,2\}} \in V(\{1,2\}), R_{3}^{x}=N$, and $x \in V(N)$. This means that $(N, V)$ is an exact partition game.

Let $\widetilde{\Gamma}_{\exp }$ denote the class of all exact partition games. In Example 4 , we observe that the equal split-off set of an exact partition game intersects the core. We show that the equal split-off set intersects the core of a game if and only if it is an exact partition game. Then Lemma 2 implies that the equal split-off set of an exact partition game is single-valued.

\section{Lemma 3}

Let $(N, V) \in \widetilde{\Gamma}_{\text {all }}$. Then $\operatorname{ESOS}(N, V) \cap C(N, V) \neq \emptyset$ if and only if $(N, V) \in \widetilde{\Gamma}_{\text {exp }}$.

Proof. Assume that there exists $x \in \operatorname{ESOS}(N, V) \cap C(N, V)$. Then $x_{R_{k}^{x}} \in \mathrm{WP}\left(V\left(R_{k}^{x}\right)\right)$ for all $k \in \mathbb{N}$. Hence, $(N, V) \in \widetilde{\Gamma}_{\text {exp }}$.

Assume that $(N, V) \in \widetilde{\Gamma}_{\text {exp }}$. Let $x \in C(N, V)$ be such that $x_{R_{k}^{x}} \in V\left(R_{k}^{x}\right)$ for all $k \in \mathbb{N}$. Then $x_{R_{k}^{x}} \in \mathrm{WP}\left(V\left(R_{k}^{x}\right)\right)$ for all $k \in \mathbb{N}$. Let $k \in \mathbb{N}$ be such that $R_{k-1}^{x} \neq N$. Then

$$
a_{k}^{x}=\max \left\{t \in \mathbb{R}_{+} \mid\left(t e_{R_{k}^{x} \backslash R_{k-1}^{x}}, x_{R_{k-1}^{x}}\right) \in V\left(R_{k}^{x}\right)\right\} .
$$

Note that $x_{i} \leq a_{k}^{x}$ for all $i \in N \backslash R_{k-1}^{x}$. Suppose for the sake of contradiction that there exists $S \in 2^{N \backslash R_{k-1}^{x}} \backslash\{\emptyset\}$ such that

$$
a_{k}^{x}<\max \left\{t \in \mathbb{R}_{+} \mid\left(t e_{S}, x_{R_{k-1}^{x}}\right) \in V\left(S \cup R_{k-1}^{x}\right)\right\} .
$$

By comprehensiveness, $x_{S \cup R_{k-1}^{x}} \in V\left(S \cup R_{k-1}^{x}\right) \backslash \mathrm{SP}\left(V\left(S \cup R_{k-1}^{x}\right)\right)$. By nonlevelness, $x_{S \cup R_{k-1}^{x}} \in V\left(S \cup R_{k-1}^{x}\right) \backslash \mathrm{WP}\left(V\left(S \cup R_{k-1}^{x}\right)\right)$. This contradicts that $x \in C(N, V)$, which implies that

$$
R_{k}^{x} \backslash R_{k-1}^{x} \in \underset{S \in 2^{N \backslash R_{k-1}^{x} \backslash\{\emptyset\}}}{\operatorname{argmax}} \max \left\{t \in \mathbb{R}_{+} \mid\left(t e_{S}, x_{R_{k-1}^{x}}\right) \in V\left(S \cup R_{k-1}^{x}\right)\right\} .
$$

Hence, $x \in \operatorname{ESOS}(N, V)$. 
The following punctual properties are satisfied by the equal split-off set on the class of exact partition games.

\section{Nonemptiness}

$\sigma(N, V) \neq \emptyset$ for all $(N, V) \in \Gamma$.

\section{Feasible richness}

$x_{R_{1}^{x}} \in V\left(R_{1}^{x}\right)$ for all $(N, V) \in \Gamma$ and all $x \in \sigma(N, V)$.

\section{Strong feasible richness}

$x_{R_{k}^{x}} \in V\left(R_{k}^{x}\right)$ for all $(N, V) \in \Gamma$, all $x \in \sigma(N, V)$, and all $k \in \mathbb{N}$.

\section{Equal payoff stability}

$x_{S} \nless\left(\max \left\{t \in \mathbb{R}_{+} \mid t e_{S} \in V(S)\right\}\right)_{i \in S}$ for all $(N, V) \in \Gamma$, all $x \in \sigma(N, V)$, and all $S \in$ $2^{N} \backslash\{\emptyset\}$.

\section{Core selection}

$\sigma(N, V) \subseteq C(N, V)$ for all $(N, V) \in \Gamma$.

Nonemptiness requires that a solution assigns to all games at least one payoff allocation. Feasible richness requires that the richest players are able to obtain their payoffs by themselves. Strong feasible richness requires that the richest players, the richest and second richest players, and so on, are able to obtain their payoffs jointly by themselves. Equal payoff stability requires that no coalition is better off by an attainable equal payoff allocation, i.e. for all coalitions there exists a member whose allocated payoff is at least the maximal attainable equal payoff. Core selection requires that only core elements are assigned. Note that strong feasible richness implies feasible richness, and core selection implies equal payoff stability. Clearly, the equal split-off set satisfies nonemptiness and strong feasible richness. By Lemma 2 and Lemma3, the equal split-off set satisfies core selection on the class of exact partition games. In fact, an axiomatic characterization of the equal split-off set in terms of nonemptiness, strong feasible richness, and core selection on the class of exact partition games is directly obtained.

\section{Theorem 1}

The equal split-off set is the unique solution for exact partition games satisfying nonemptiness, strong feasible richness, and core selection.

Proof. Clearly, the equal split-off set satisfies nonemptiness and strong feasible richness. By Lemma 2 and Lemma 3 , the equal split-off set satisfies core selection on $\widetilde{\Gamma}_{\text {exp }}$.

To prove uniqueness, let $\sigma$ be a solution on $\widetilde{\Gamma}_{\text {exp }}$ satisfying nonemptiness, strong feasible richness, and core selection. Let $(N, V) \in \widetilde{\Gamma}_{\text {exp }}$. By nonemptiness, there exists $x \in \sigma(N, V)$. By core selection and strong feasible richness, $x \in C(N, v)$ and $x_{R_{k}^{x}} \in V\left(R_{k}^{x}\right)$ for all $k \in \mathbb{N}$. By the proof of Lemma 3 this implies that $x \in \operatorname{ESOS}(N, V)$. By Lemma $2|\operatorname{ESOS}(N, V)|=1$. Hence, $\sigma(N, V)=\operatorname{ESOS}(N, V)$. 
Theorem 1 generalizes the corresponding result of Calleja et al. (2021) for transferable utility games. The empty solution, which assigns to all exact partition games the empty set, satisfies strong feasible richness and core selection, but does not satisfy nonemptiness. The core satisfies nonemptiness and core selection, but does not satisfy strong feasible richness. The equal payoff solution, which assigns to all exact partition games the maximal attainable equal payoff allocation of the grand coalition, satisfies nonemptiness and strong feasible richness, but does not satisfy core selection. Hence, the properties in Theorem 1 are independent.

The equal split-off set is not the unique solution for exact partition games satisfying nonemptiness, feasible richness, and equal payoff stability. The solution which assigns $(6,2,2)$ to the game in Example 4, and the equal split-off set to all other exact partition games, also satisfies these properties. However, this solution does not apply feasible richness in a coherent way to nontransferable utility games with variable population. In other words, it does not satisfy the relational property of consistency. Suppose that we apply a certain solution to select payoff allocations for the grand coalition and consider one such assigned payoff allocation. Some players leave with their payoffs and the remaining players reevaluate their payoffs on the basis of a reduced game. The solution is consistent if it assigns the same payoffs to the remaining players in the reduced game as in the original game.

Peleg (1985) axiomatically characterized the core for nontransferable utility games using the consistency property where the attainable payoff allocations for the remaining players in the reduced game are the attainable payoff allocations in coalition with any subgroup of departed players in the original game when these departed players are assigned their original payoffs. This generalizes the consistency property for transferable utility games of Davis and Maschler (1965) and we refer to it as max-consistency. In order to axiomatically characterize the equal split-off set for exact partition games, we use the weaker version which only requires consistent payoff allocations when all richest players leave, to which we refer as rich-restricted max-consistency.

\section{Rich-restricted max-consistency}

$\left(N \backslash R_{1}^{x}, V_{\max }^{x}\right) \in \Gamma$ and $x_{N \backslash R_{1}^{x}} \in \sigma\left(N \backslash R_{1}^{x}, V_{\max }^{x}\right)$ for all $(N, V) \in \Gamma$ and all $x \in \sigma(N, V)$ with $R_{1}^{x} \neq N$, where

$$
V_{\text {max }}^{x}(S)= \begin{cases}\left\{y_{N \backslash R_{1}^{x}} \in \mathbb{R}_{+}^{N \backslash R_{1}^{x}} \mid\left(y_{N \backslash R_{1}^{x}}, x_{R_{1}^{x}}\right) \in V(N)\right\} & \text { if } S=N \backslash R_{1}^{x} ; \\ \bigcup_{Q \subseteq R_{1}^{x}}\left\{y_{S} \in \mathbb{R}_{+}^{S} \mid\left(y_{S}, x_{Q}\right) \in V(S \cup Q)\right\} & \text { if } \emptyset \subset S \subset N \backslash R_{1}^{x} .\end{cases}
$$

\section{Theorem 2}

The equal split-off set is the unique solution for exact partition games satisfying nonemptiness, feasible richness, equal payoff stability, and rich-restricted max-consistency. 
Proof. Clearly, the equal split-off set satisfies nonemptiness and feasible richness. By Lemma 2 and Lemma 3 , the equal split-off set satisfies equal payoff stability on $\widetilde{\Gamma}_{\text {exp }}$. To show that the equal split-off set satisfies rich-restricted max-consistency on $\widetilde{\Gamma}_{\text {exp }}$, let $(N, V) \in \widetilde{\Gamma}_{\text {exp }}$ and let $x \in \operatorname{ESOS}(N, V)$ be such that $R_{1}^{x} \neq N$. By Lemma 2 and Lemma $3, x \in C(N, V)$. Suppose for the sake of contradiction that $x_{N \backslash R_{1}^{x}} \notin C\left(N \backslash R_{1}^{x}, V_{\text {max }}^{x}\right)$. Then there exist $S \in 2^{N}$ with $\emptyset \subset S \subset N \backslash R_{1}^{x}$ and $y_{S} \in V_{\text {max }}^{x}(S)$ such that $y_{S}>x_{S}$. This implies that there exists $Q \subseteq R_{1}^{x}$ such that $\left(y_{S}, x_{Q}\right) \in V(S \cup Q)$. By comprehensiveness, $x_{S \cup Q} \in$ $V(S \cup Q) \backslash \mathrm{SP}(V(S \cup Q))$. By nonlevelness, $x_{S \cup Q} \in V(S \cup Q) \backslash \mathrm{WP}(V(S \cup Q))$, which implies that there exists $z_{S \cup Q} \in V(S \cup Q)$ such that $z_{S \cup Q}>x_{S \cup Q}$. This contradicts that $x \in C(N, V)$, so $x_{N \backslash R_{1}^{x}} \in C\left(N \backslash R_{1}^{x}, V_{\text {max }}^{x}\right)$. Note that $R_{k}^{x_{N} \backslash R_{1}^{x}}=R_{k+1}^{x} \backslash R_{1}^{x}$ for all $k \in \mathbb{N}$. For all $k \in \mathbb{N}, x_{R_{k+1}^{x}} \in V\left(R_{k+1}^{x}\right)$, so $\left(x_{R_{k} x_{N} \backslash R_{1}^{x}}, x_{R_{1}^{x}}\right) \in V\left(R_{k}^{x_{N} \backslash R_{1}^{x}} \cup R_{1}^{x}\right)$, which implies

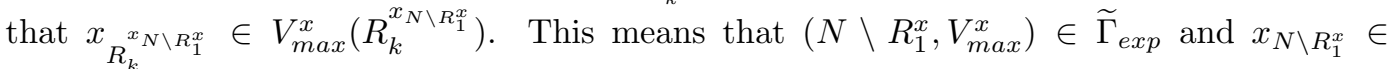
$\operatorname{ESOS}\left(N \backslash R_{1}^{x}, V_{\text {max }}^{x}\right)$. Hence, the equal split-off set satisfies rich-restricted max-consistency.

To prove uniqueness, let $\sigma$ be a solution on $\widetilde{\Gamma}_{\text {exp }}$ satisfying nonemptiness, feasible richness, equal payoff stability, and rich-restricted max-consistency. We show by induction on the number of players that $\sigma(N, V)$ consists of one uniquely defined payoff allocation for all $(N, V) \in \widetilde{\Gamma}_{\text {exp }}$. By nonemptiness and equal payoff stability, $\sigma(N, V)=\{\mathrm{WP}(V(N))\}$ for all $(N, V) \in \widetilde{\Gamma}_{\text {exp }}$ with $|N|=1$. Let $k \in \mathbb{N}$ and assume that $\sigma(N, V)$ consists of one uniquely defined payoff allocation for all $(N, V) \in \widetilde{\Gamma}_{e x p}$ with $|N| \leq k$. Let $(N, V) \in \widetilde{\Gamma}_{e x p}$ with $|N|=k+1$. By nonemptiness, there exists $x \in \sigma(N, V)$. By equal payoff stability,

$$
a_{1}^{x} \geq \max _{S \in 2^{N} \backslash\{\emptyset\}} \max \left\{t \in \mathbb{R}_{+} \mid t e_{S} \in V(S)\right\} \geq \max \left\{t \in \mathbb{R}_{+} \mid t e_{R_{1}^{x}} \in V\left(R_{1}^{x}\right)\right\} .
$$

By feasible richness,

$$
a_{1}^{x} \leq \max \left\{t \in \mathbb{R}_{+} \mid t e_{R_{1}^{x}} \in V\left(R_{1}^{x}\right)\right\} \leq \max _{S \in 2^{N} \backslash\{\emptyset\}} \max \left\{t \in \mathbb{R}_{+} \mid t e_{S} \in V(S)\right\} .
$$

This implies that

$$
\begin{aligned}
a_{1}^{x}=\max _{S \in 2^{N} \backslash\{\emptyset\}} \max \left\{t \in \mathbb{R}_{+} \mid t e_{S} \in V(S)\right\} \\
\text { and } \quad R_{1}^{x} \in \underset{S \in 2^{N} \backslash\{\emptyset\}}{\operatorname{argmax}} \max \left\{t \in \mathbb{R}_{+} \mid t e_{S} \in V(S)\right\} .
\end{aligned}
$$

Define $U^{V}=\bigcup \operatorname{argmax}_{S \in 2^{N} \backslash\{\emptyset\}} \max \left\{t \in \mathbb{R}_{+} \mid t e_{S} \in V(S)\right\}$. Since $(N, V) \in \widetilde{\Gamma}_{\text {exp }}$, Lemma 2 and Lemma 3 imply that $U^{V} \in \operatorname{argmax}_{S \in 2^{N} \backslash\{\emptyset\}} \max \left\{t \in \mathbb{R}_{+} \mid t e_{S} \in V(S)\right\}$. Suppose for the sake of contradiction that $R_{1}^{x} \neq U^{V}$. By rich-restricted max-consistency, $x_{N \backslash R_{1}^{x}} \in$ $\sigma\left(N \backslash R_{1}^{x}, V_{\max }^{x}\right)$. 
By equal payoff stability, there exists $i \in U^{V} \backslash R_{1}^{x}$ such that

$$
\begin{aligned}
x_{i} & \geq \max \left\{t \in \mathbb{R}_{+} \mid t e_{U^{V} \backslash R_{1}^{x}} \in V_{\max }^{x}\left(U^{V} \backslash R_{1}^{x}\right)\right\} \\
& \geq \max \left\{t \in \mathbb{R}_{+} \mid\left(t e_{U^{V} \backslash R_{1}^{x}}, x_{R_{1}^{x}}\right) \in V\left(U^{V}\right)\right\} \\
& =\max \left\{t \in \mathbb{R}_{+} \mid\left(t e_{U^{V} \backslash R_{1}^{x}}, a_{1}^{x} e_{R_{1}^{x}}\right) \in V\left(U^{V}\right)\right\} \\
& =a_{1}^{x},
\end{aligned}
$$

where the last equality follows from nonlevelness. This is a contradiction, so $R_{1}^{x}=U^{V}$. If $R_{1}^{x}=U^{V}=N$, then $\sigma(N, V)=\left\{\left(\max \left\{t \in \mathbb{R}_{+} \mid t e_{N} \in V(N)\right\}\right)_{i \in N}\right\}$. Suppose that $R_{1}^{x}=U^{V} \neq N$. By rich-restricted max-consistency, $x_{N \backslash R_{1}^{x}} \in \sigma\left(N \backslash R_{1}^{x}, V_{\text {max }}^{x}\right)$, where, by the induction hypothesis, $\sigma\left(N \backslash R_{1}^{x}, V_{\max }^{x}\right)$ consists of one uniquely defined payoff allocation since $\left|N \backslash R_{1}^{x}\right| \leq k$. Hence, $\sigma(N, V)$ consists of one uniquely defined payoff allocation.

Theorem 2 generalizes the corresponding result of Dietzenbacher and Yanovskaya (2021) for transferable utility games. The empty solution, which assigns to all exact partition games the empty set, satisfies feasible richness, equal payoff stability, and rich-restricted max-consistency, but does not satisfy nonemptiness. The solution which assigns the core to all exact partition games with at most two players, and the equal split-off set to all exact partition games with more players, satisfies nonemptiness, equal payoff stability, and richrestricted max-consistency, but does not satisfy feasible richness. The equal payoff solution, which assigns to all exact partition games the maximal attainable equal payoff allocation of the grand coalition, satisfies nonemptiness, feasible richness, and rich-restricted maxconsistency, but does not satisfy equal payoff stability. The solution which assigns $(6,2,2)$ to the game in Example 4 and the equal split-off set to all other exact partition games, satisfies nonemptiness, feasible richness, and equal payoff stability, but does not satisfy rich-restricted max-consistency. Hence, the properties in Theorem 2 are independent.

Tadenuma (1992) axiomatically characterized the core for nontransferable utility games using the alternative consistency property where the attainable payoff allocations for the remaining players in the reduced game are the attainable payoff allocations in coalition with all departed players in the original game when these departed players are assigned their original payoffs. We refer to this consistency property as complement-consistency. In order to axiomatically characterize the equal split-off set for exact partition games, we use the weaker version which only requires consistent payoff allocations when all richest players leave, to which we refer as rich-restricted complement consistency.

\section{Rich-restricted complement-consistency}

$\left(N \backslash R_{1}^{x}, V_{\text {comp }}^{x}\right) \in \Gamma$ and $x_{N \backslash R_{1}^{x}} \in \sigma\left(N \backslash R_{1}^{x}, V_{\text {comp }}^{x}\right)$ for all $(N, V) \in \Gamma$ and all $x \in \sigma(N, V)$ with $R_{1}^{x} \neq N$, where for all $S \in 2^{N \backslash R_{1}^{x}} \backslash\{\emptyset\}$,

$$
V_{\text {comp }}^{x}(S)=\left\{y_{S} \in \mathbb{R}_{+}^{S} \mid\left(y_{S}, x_{R_{1}^{x}}\right) \in V\left(S \cup R_{1}^{x}\right)\right\} .
$$




\section{Theorem 3}

The equal split-off set is the unique solution for exact partition games satisfying nonemptiness, feasible richness, equal payoff stability, and rich-restricted complement-consistency.

Proof. Clearly, the equal split-off set satisfies nonemptiness and feasible richness. By Lemma 2 and Lemma 3 the equal split-off set satisfies equal payoff stability on $\widetilde{\Gamma}_{\text {exp }}$. To show that the equal split-off set satisfies rich-restricted complement-consistency on $\widetilde{\Gamma}_{\text {exp }}$, let $(N, V) \in \widetilde{\Gamma}_{\text {exp }}$ and let $x \in \operatorname{ESOS}(N, V)$ be such that $R_{1}^{x} \neq N$. By Lemma 2 and Lemma $3, x \in C(N, V)$. Suppose for the sake of contradiction that $x_{N \backslash R_{1}^{x}} \notin C\left(N \backslash R_{1}^{x}, V_{\text {comp }}^{x}\right)$. Then there exist $S \in 2^{N}$ with $\emptyset \subset S \subset N \backslash R_{1}^{x}$ and $y_{S} \in V_{\text {comp }}^{x}(S)$ such that $y_{S}>x_{S}$. This implies that $\left(y_{S}, x_{R_{1}^{x}}\right) \in V\left(S \cup R_{1}^{x}\right)$. By comprehensiveness, $x_{S \cup R_{1}^{x}} \in V\left(S \cup R_{1}^{x}\right) \backslash \mathrm{SP}\left(V\left(S \cup R_{1}^{x}\right)\right)$. By nonlevelness, $x_{S \cup R_{1}^{x}} \in V\left(S \cup R_{1}^{x}\right) \backslash \mathrm{WP}\left(V\left(S \cup R_{1}^{x}\right)\right)$, which implies that there exists $z_{S \cup R_{1}^{x}} \in V\left(S \cup R_{1}^{x}\right)$ such that $z_{S \cup R_{1}^{x}}>x_{S \cup R_{1}^{x}}$. This contradicts that $x \in C(N, V)$, so $x_{N \backslash R_{1}^{x}} \in C\left(N \backslash R_{1}^{x}, V_{\text {comp }}^{x}\right)$. Note that $R_{k}^{x_{N \backslash R_{1}^{x}}}=R_{k+1}^{x} \backslash R_{1}^{x}$ for all $k \in \mathbb{N}$. For all $k \in \mathbb{N}$, $x_{R_{k+1}^{x}} \in V\left(R_{k+1}^{x}\right)$, so $\left(x_{R_{k}^{x_{N} \backslash R_{1}^{x}}}, x_{R_{1}^{x}}\right) \in V\left(R_{k}^{x_{N} \backslash R_{1}^{x}} \cup R_{1}^{x}\right)$, which implies that $x_{R_{k}^{x_{N} \backslash R_{1}^{x}}} \in$ $V_{\text {comp }}^{x}\left(R_{k}^{x_{N} \backslash R_{1}^{x}}\right)$. This means that $\left(N \backslash R_{1}^{x}, V_{\text {comp }}^{x}\right) \in \widetilde{\Gamma}_{\text {exp }}$ and $x_{N \backslash R_{1}^{x}} \in \operatorname{ESOS}\left(N \backslash R_{1}^{x}, V_{\text {comp }}^{x}\right)$. Hence, the equal split-off set satisfies rich-restricted complement-consistency.

To prove uniqueness, let $\sigma$ be a solution on $\widetilde{\Gamma}_{\text {exp }}$ satisfying nonemptiness, feasible richness, equal payoff stability, and rich-restricted complement-consistency. We show by induction on the number of players that $\sigma(N, V)$ consists of one uniquely defined payoff allocation for all $(N, V) \in \widetilde{\Gamma}_{\text {exp }}$. By nonemptiness and equal payoff stability, $\sigma(N, V)=\{\mathrm{WP}(V(N))\}$ for all $(N, V) \in \widetilde{\Gamma}_{\text {exp }}$ with $|N|=1$. Let $k \in \mathbb{N}$ and assume that $\sigma(N, V)$ consists of one uniquely defined payoff allocation for all $(N, V) \in \widetilde{\Gamma}_{\text {exp }}$ with $|N| \leq k$. Let $(N, V) \in \widetilde{\Gamma}_{\text {exp }}$ with $|N|=k+1$. By nonemptiness, there exists $x \in \sigma(N, V)$. By equal payoff stability,

$$
a_{1}^{x} \geq \max _{S \in 2^{N} \backslash\{\emptyset\}} \max \left\{t \in \mathbb{R}_{+} \mid t e_{S} \in V(S)\right\} \geq \max \left\{t \in \mathbb{R}_{+} \mid t e_{R_{1}^{x}} \in V\left(R_{1}^{x}\right)\right\} .
$$

By feasible richness,

$$
a_{1}^{x} \leq \max \left\{t \in \mathbb{R}_{+} \mid t e_{R_{1}^{x}} \in V\left(R_{1}^{x}\right)\right\} \leq \max _{S \in 2^{N} \backslash\{\emptyset\}} \max \left\{t \in \mathbb{R}_{+} \mid t e_{S} \in V(S)\right\} .
$$

This implies that

$$
\begin{aligned}
a_{1}^{x}=\max _{S \in 2^{N} \backslash\{\emptyset\}} \max \left\{t \in \mathbb{R}_{+} \mid t e_{S} \in V(S)\right\} \\
\text { and } \quad R_{1}^{x} \in \underset{S \in 2^{N} \backslash\{\emptyset\}}{\operatorname{argmax}} \max \left\{t \in \mathbb{R}_{+} \mid t e_{S} \in V(S)\right\} .
\end{aligned}
$$

Define $U^{V}=\bigcup \operatorname{argmax}_{S \in 2^{N} \backslash\{\emptyset\}} \max \left\{t \in \mathbb{R}_{+} \mid t e_{S} \in V(S)\right\}$. Since $(N, V) \in \widetilde{\Gamma}_{e x p}$, Lemma 2 and Lemma 3 imply that $U^{V} \in \operatorname{argmax}_{S \in 2^{N} \backslash\{\emptyset\}} \max \left\{t \in \mathbb{R}_{+} \mid t e_{S} \in V(S)\right\}$. 
Suppose for the sake of contradiction that $R_{1}^{x} \neq U^{V}$. By rich-restricted complementconsistency, $x_{N \backslash R_{1}^{x}} \in \sigma\left(N \backslash R_{1}^{x}, V_{\text {comp }}^{x}\right)$. By equal payoff stability, there exists $i \in U^{V} \backslash R_{1}^{x}$ such that

$$
\begin{aligned}
x_{i} & \geq \max \left\{t \in \mathbb{R}_{+} \mid t e_{U^{V} \backslash R_{1}^{x}} \in V_{\text {comp }}^{x}\left(U^{V} \backslash R_{1}^{x}\right)\right\} \\
& =\max \left\{t \in \mathbb{R}_{+} \mid\left(t e_{U^{V} \backslash R_{1}^{x}}, x_{R_{1}^{x}}\right) \in V\left(U^{V}\right)\right\} \\
& =\max \left\{t \in \mathbb{R}_{+} \mid\left(t e_{U^{V} \backslash R_{1}^{x}}, a_{1}^{x} e_{R_{1}^{x}}\right) \in V\left(U^{V}\right)\right\} \\
& =a_{1}^{x},
\end{aligned}
$$

where the last equality follows from nonlevelness. This is a contradiction, so $R_{1}^{x}=U^{V}$. If $R_{1}^{x}=U^{V}=N$, then $\sigma(N, V)=\left\{\left(\max \left\{t \in \mathbb{R}_{+} \mid t e_{N} \in V(N)\right\}\right)_{i \in N}\right\}$. Suppose that $R_{1}^{x}=$ $U^{V} \neq N$. By rich-restricted complement-consistency, $x_{N \backslash R_{1}^{x}} \in \sigma\left(N \backslash R_{1}^{x}, V_{\text {comp }}^{x}\right)$, where, by the induction hypothesis, $\sigma\left(N \backslash R_{1}^{x}, V_{\text {comp }}^{x}\right)$ consists of one uniquely defined payoff allocation since $\left|N \backslash R_{1}^{x}\right| \leq k$. Hence, $\sigma(N, V)$ consists of one uniquely defined payoff allocation.

Theorem 3 generalizes the corresponding result of Dietzenbacher and Yanovskaya (2021) for transferable utility games. The empty solution satisfies feasible richness, equal payoff stability, and rich-restricted complement-consistency, but does not satisfy nonemptiness. The solution which assigns the core to all exact partition games with at most two players, and the equal split-off set to all exact partition games with more players, satisfies nonemptiness, equal payoff stability, and rich-restricted complement-consistency, but does not satisfy feasible richness. The equal payoff solution satisfies nonemptiness, feasible richness, and richrestricted complement-consistency, but does not satisfy equal payoff stability. The solution which assigns $(6,2,2)$ to the game in Example 4 , and the equal split-off set to all other exact partition games, satisfies nonemptiness, feasible richness, and equal payoff stability, but does not satisfy rich-restricted complement-consistency. Hence, the properties in Theorem 3 are independent.

\section{$5 \quad$ Bargaining and bankruptcy problems}

In this section, we study the equal split-off set for the class of bargaining problems and the class of bankruptcy problems with nontransferable utility. A bargaining problem (cf. Nash 1950) is a pair $(N, F)$ where $N$ is a nonempty and finite set of agents and $F \subseteq \mathbb{R}_{+}^{N}$ is a nonempty, closed, bounded, comprehensive, and nonleveled set of attainable payoff allocations. 
The bargaining game $\left(N, V^{F}\right) \in \widetilde{\Gamma}_{\text {all }}$ corresponding to the bargaining problem $(N, F)$ is the nontransferable utility game defined by

$$
V^{F}(S)= \begin{cases}F & \text { if } S=N ; \\ \left\{x_{S} \in \mathbb{R}_{+}^{S} \mid x_{S} \leq 0_{S}\right\} & \text { if } \emptyset \subset S \subset N .\end{cases}
$$

The core of a bargaining game $\left(N, V^{F}\right)$ is given by $C\left(N, V^{F}\right)=\mathrm{WP}(F)$. We show that bargaining games are exact partition games and provide an explicit expression of the equal split-off set.

\section{Theorem 4}

Let $(N, F)$ be a bargaining problem. Then $\left(N, V^{F}\right) \in \widetilde{\Gamma}_{\text {exp }}$ and

$$
\operatorname{ESOS}\left(N, V^{F}\right)=\lambda^{F} e_{N}
$$

where $\lambda^{F}=\max \left\{t \in \mathbb{R}_{+} \mid t e_{N} \in F\right\}$.

Proof. Let $x \in \mathbb{R}_{+}^{N}$ be defined by $x_{i}=\lambda^{F}$ for all $i \in N$. Then $x \in \mathrm{WP}(F)$, which implies that $x \in C\left(N, V^{F}\right)$. Moreover, $R_{k}^{x}=N$ and $x_{R_{k}^{x}} \in V\left(R_{k}^{x}\right)$ for all $k \in \mathbb{N}$. Hence, $\left(N, V^{F}\right) \in \widetilde{\Gamma}_{e x p}$ and $\operatorname{ESOS}\left(N, V^{F}\right)=x$.

Theorem 4 implies that the equal split-off set for bargaining games coincides with the Kalai (1977) solution for bargaining problems. This is illustrated by the following example.

\section{Example 5}

Let $(N, F)$ be the bargaining problem with $N=\{1,2\}$ and

$$
F=\left\{x \in \mathbb{R}_{+}^{N} \mid x_{1}^{2}+9 x_{2} \leq 36\right\} .
$$

The equal split-off set is $\operatorname{ESOS}\left(N, V^{F}\right)=\{(3,3)\}$.

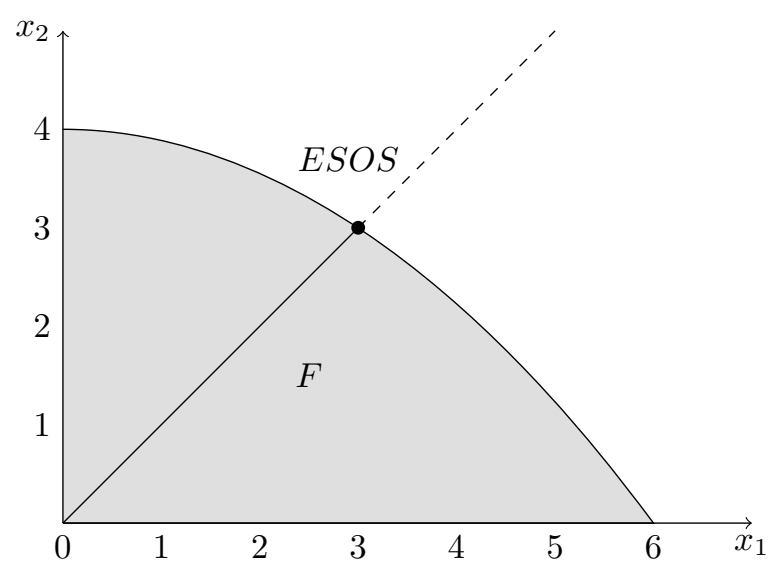


A bankruptcy problem (cf. Orshan et al. 2003), or bargaining problem with claims (cf. Chun and Thomson 1992), is a triple $(N, E, c)$ where $N$ is a nonempty and finite set of claimants, $E \subseteq \mathbb{R}_{+}^{N}$ is a nonempty, closed, bounded, comprehensive, and nonleveled set of attainable payoff allocations, and $c \in \mathbb{R}_{+}^{N} \backslash E$ is a vector of claims of $N$ on $E$.

The bankruptcy game (cf. Dietzenbacher 2018) $\left(N, V^{E, c}\right) \in \widetilde{\Gamma}_{\text {all }}$ corresponding to the bankruptcy problem $(N, E, c)$ is the nontransferable utility game defined by

$$
V^{E, c}(S)= \begin{cases}\left\{x_{S} \in \mathbb{R}_{+}^{S} \mid\left(x_{S}, c_{N \backslash S}\right) \in E\right\} & \text { if }\left(0_{S}, c_{N \backslash S}\right) \in E ; \\ \left\{x_{S} \in \mathbb{R}_{+}^{S} \mid x_{S} \leq 0_{S}\right\} & \text { if }\left(0_{S}, c_{N \backslash S}\right) \notin E .\end{cases}
$$

The core of a bankruptcy game $\left(N, V^{E, c}\right)$ is given by $C\left(N, V^{E, c}\right)=\{x \in \mathrm{WP}(E) \mid x \leq c\}$. We show that bankruptcy games are exact partition games and provide an explicit expression of the equal split-off set.

\section{Theorem 5}

Let $(N, E, c)$ be a bankruptcy problem. Then $\left(N, V^{E, c}\right) \in \widetilde{\Gamma}_{\exp }$ and

$$
\operatorname{ESOS}\left(N, V^{E, c}\right)=\left(\min \left\{\lambda^{E, c}, c_{i}\right\}\right)_{i \in N},
$$

where $\lambda^{E, c}=\max \left\{t \in \mathbb{R}_{+} \mid\left(\min \left\{t, c_{i}\right\}\right)_{i \in N} \in E\right\}$.

Proof. Let $x \in \mathbb{R}_{+}^{N}$ be defined by $x_{i}=\min \left\{\lambda^{E, c}, c_{i}\right\}$ for all $i \in N$. Then $x \leq c$ and by nonlevelness, $x \in \mathrm{WP}(E)$. This implies that $x \in C\left(N, V^{E, c}\right)$. Let $k \in \mathbb{N}$ be such that $R_{k-1}^{x} \neq N$. If $x_{i}=\lambda^{E, c}$ for all $i \in R_{k}^{x} \backslash R_{k-1}^{x}$, then $R_{k-1}^{x}=\emptyset$ and $x_{R_{k}^{x}} \in V\left(R_{k}^{x}\right)$. If $x_{i}=c_{i}$ for all $i \in R_{k}^{x} \backslash R_{k-1}^{x}$, then $x_{i}=c_{i}$ for all $i \in N \backslash R_{k}^{x}$ and $x_{R_{k}^{x}} \in V\left(R_{k}^{x}\right)$. Hence, $\left(N, V^{E, c}\right) \in \widetilde{\Gamma}_{e x p}$ and $\operatorname{ESOS}\left(N, V^{E, c}\right)=x$.

Theorem 5 implies that the equal split-off set for bankruptcy games coincides with the constrained equal awards rule, the constrained Kalai solution (cf. Albizuri et al. 2020) for bankruptcy problems. This illustrated by the following example.

\section{Example 6}

Let $(N, E, c)$ be the bankruptcy problem with $N=\{1,2\}, c=(2,5)$, and

$$
E=\left\{x \in \mathbb{R}_{+}^{N} \mid x_{1}^{2}+9 x_{2} \leq 36\right\} .
$$

The corresponding bankruptcy game is given by

$$
V^{E, c}(S)= \begin{cases}\left\{x_{1} \in \mathbb{R}_{+}^{\{1\}} \mid x_{1} \leq 0\right\} & \text { if } S=\{1\} ; \\ \left\{x_{2} \in \mathbb{R}_{+}^{\{2\}} \mid x_{2} \leq 3 \frac{5}{9}\right\} & \text { if } S=\{2\} ; \\ E & \text { if } S=N .\end{cases}
$$


The equal split-off set is $\operatorname{ESOS}\left(N, V^{E, c}\right)=\left\{\left(2,3 \frac{5}{9}\right)\right\}$ corresponding to ordered partition $\{\{2\},\{1\}\}$.

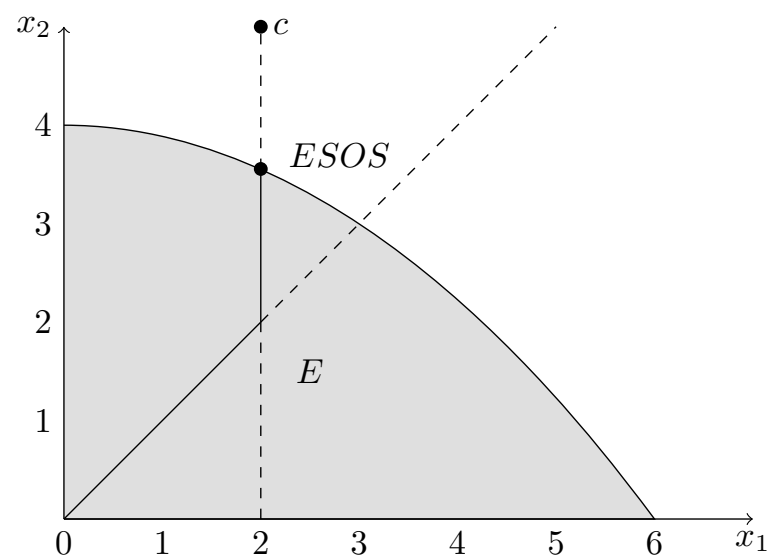

\section{Acknowledgements}

Support from the HSE University Basic Research Program is gratefully acknowledged.

\section{References}

Albizuri, M., B. Dietzenbacher, and J. Zarzuelo (2020). Bargaining with independence of higher or irrelevant claims. Journal of Mathematical Economics, 91, 11-17.

Asscher, N. (1976). An ordinal bargaining set for games without side payments. Mathematics of Operations Research, 1(4), 381-389.

Asscher, N. (1977). A cardinal bargaining set for games without side payments. International Journal of Game Theory, 6(2), 87-114.

Aumann, R. (1985a). An axiomatization of the non-transferable utility value. Econometrica, 53(3), 599-612.

Aumann, R. (1985b). On the non-transferable utility value: a comment on the Roth-Shafer examples. Econometrica, 53(3), 667-678.

Aumann, R. (1986). On the non-transferable utility value: rejoinder. Econometrica, 54(4), 985-989.

Branzei, R., D. Dimitrov, and S. Tijs (2006). The equal split-off set for cooperative games. Banach Center Publications, 71, 39-46.

Calleja, P., F. Llerena, and P. Sudhölter (2021). Axiomatizations of Dutta-Ray's egalitarian solution on the domain of convex games. Journal of Mathematical Economics, 95, 102477 . 
Chun, Y. and W. Thomson (1992). Bargaining problems with claims. Mathematical Social Sciences, 24(1), 19-33.

Clippel, G. de, H. Peters, and H. Zank (2004). Axiomatizing the Harsanyi solution, the symmetric egalitarian solution and the consistent solution for NTU-games. International Journal of Game Theory, 33(1), 145-158.

Davis, M. and M. Maschler (1965). The kernel of a cooperative game. Naval Research Logistics Quarterly, 12(3), 223-259.

Dietzenbacher, B. (2018). Bankruptcy games with nontransferable utility. Mathematical Social Sciences, 92, 16-21.

Dietzenbacher, B. and E. Yanovskaya (2021). Consistency of the equal split-off set. International Journal of Game Theory, 50(1), 1-22.

Dutta, B. and D. Ray (1989). A concept of egalitarianism under participation constraints. Econometrica, 57(3), 615-635.

Greenberg, J. (1985). Cores of convex games without side payments. Mathematics of Operations Research, 10(3), 523-525.

Harsanyi, J. (1980). Comments on Roth's paper, 'Values for games without side payments'. Econometrica, 48(2), 477.

Hart, S. (1985a). An axiomatization of Harsanyi's nontransferable utility solution. Econometrica, 53(6), 1295-1313.

Hart, S. (1985b). Nontransferable utility games and markets: some examples and the Harsanyi solution. Econometrica, 53(6), 1445-1450.

Hart, S. and M. Kurz (1983). Endogenous formation of coalitions. Econometrica, 51(4), 1047-1064.

Hart, S. and A. Mas-Colell (1996). Bargaining and value. Econometrica, 64(2), 357-380.

Hendrickx, R., P. Borm, and J. Timmer (2002). A note on NTU convexity. International Journal of Game Theory, 31(1), 29-37.

Kalai, E. (1977). Proportional solutions to bargaining situations: interpersonal utility comparisons. Econometrica, 45(7), 1623-1630.

Kalai, E. and D. Samet (1985). Monotonic solutions to general cooperative games. Econometrica, 53(2), 307-327.

Lejano, R. (2011). A note on solution concepts for nontransferable utility games. Journal of Mathematical Economics, 47(6), 777-780.

Llerena, F. and L. Mauri (2017). On the existence of the Dutta-Ray's egalitarian solution. Mathematical Social Sciences, 89, 92-99.

Nash, J. (1950). The bargaining problem. Econometrica, 18(2), 155-162. 
Orshan, G., F. Valenciano, and J. Zarzuelo (2003). The bilateral consistent prekernel, the core, and NTU bankruptcy problems. Mathematics of Operations Research, 28(2), $268-282$.

Otten, G., P. Borm, B. Peleg, and S. Tijs (1998). The MC-value for monotonic NTUgames. International Journal of Game Theory, 27(1), 37-47.

Peleg, B. (1985). An axiomatization of the core of cooperative games without side payments. Journal of Mathematical Economics, 14(2), 203-214.

Roth, A. (1980). Values for games without sidepayments: some difficulties with current concepts. Econometrica, 48(2), 457-465.

Roth, A. (1986). On the non-transferable utility value: a reply to Aumann. Econometrica, 54(4), 981-984.

Samet, D. (1985). An axiomatization of the egalitarian solutions. Mathematical Social Sciences, 9(2), 173-181.

Shafer, W. (1980). On the existence and interpretation of value allocation. Econometrica, 48(2), 467-476.

Shapley, L. (1953). A value for $n$-person games. In: H. Kuhn and A. Tucker (Eds.), Contributions to the Theory of Games II, pp. 307-317. Princeton University Press.

Tadenuma, K. (1992). Reduced games, consistency, and the core. International Journal of Game Theory, 20(4), 325-334.

Yanovskaya, E. (2010). The extension of Dutta-Ray's solution to convex NTU games. International Game Theory Review, 12(4), 339-361. 